\title{
Independent cycles and chorded cycles in graphs
}

\author{
Ronald J. Gould, Kazuhide Hirohata and Paul Horn
}

In this paper, we investigate sufficient conditions on the neighborhood of independent vertices which imply that a graph contains $k$ independent cycles or chorded cycles. This is related to several results of Corrádi and Hajnal, Justesen, Wang, and Faudree and Gould on graphs containing $k$ independent cycles, and Finkel on graphs containing $k$ chorded cycles. In particular, we establish that if independent vertices in $G$ have neighborhood union at least $2 k+1$, then $G$ has $k$ chorded cycles, so long as $|G|>30 k$, and settling a conjecture of and improving a result of Faudree and Gould, who establish that $3 k$ suffices. Additionally, we show that a graph with neighborhood union of independent vertices at least $4 k+1$ has at least $k$ chorded cycles; Finkel previously established that minimum degree $3 k$ was also a sufficient condition for this.

In 1963, Corrádi and Hajnal [1] verified a conjecture of Erdős by showing that every graph $G$ on at least $3 k$ vertices with minimum degree $2 k$ contains $k$ independent cycles. In the nearly fifty years since, this result has been generalized in a number of different ways.

In 1989, Justesen [4] showed that the minimum degree condition can be replaced by a condition on the sums of degrees of non-adjacent vertices. This was improved to a sharp result by Wang [5] who showed that the condition that the minimum degree was at least $2 k$ could be replaced by the condition that the sum of degrees of non-adjacent vertices was at least $4 k-1$. Corrádi and Hajnal's result was generalized in a different direction by Finkel [3] who showed that if $G$ were a graph on at least $4 k$ vertices with minimum degree at least $3 k$, then $G$ contains at least $k$ independent chorded cycles.

In a different direction, J. Faudree and Gould [2] showed that the condition on minimum degrees in the Corrádi-Hajnal theorem could be replaced by a condition on the neighborhood of non-adjacent vertices. In particular, they showed that if $G$ is a graph on at least $3 k$ vertices such that the neighborhood of any two non-adjacent vertices has size at least $3 k$, then $G$ contains $k$ independent cycles. This result is sharp in the sense that requiring a neighborhood of size $3 k-1$ is insufficient for $k=1$. It was conjectured 
in [2], however, that it might be possible to improve their condition to one of the form $2 k+O(1)$.

In this paper, we consider both the problem of finding independent cycles and independent chorded cycles in graphs under conditions on the neighborhood union of non-adjacent vertices. Before we state the main theorems, let us briefly introduce some notation. We indicate by $N(x)$ the set of neighbors of $x$, and $N[x]=N(x) \cup\{x\}$ denotes the closed neighborhood. For a set $S \subseteq V$, we denote by $N_{S}(x)=N(x) \cap S$. For a pair of non-adjacent vertices $x, y$, we let $N(x, y)=N(x) \cup N(y)$ and define $N_{S}(x, y)$ in the obvious fashion. We define $x \sim y$ to mean that $x$ is adjacent to $y$.

We begin by generalizing the results of Finkel as follows:

Theorem 1. Suppose $G$ is a graph on at least $4 k$ vertices, such that any pair of non-adjacent vertices $x, y$ has $|N(x, y)| \geq 4 k+1$. Then there exists $k$ independent chorded cycles in $G$.

Theorem 1 is sharp in the sense that it does not hold if the condition $|N(x, y)| \geq 4 k+1$ is replaced by $|N(x, y)| \geq 4 k$. Here it fails even when $k=1$ : consider a collection of $t$ independent $K_{3}$ 's; then the neighborhood of any two non-adjacent vertices is 4 , but there are no chorded cycles. If one prefers a connected counterexample, one may append a $K_{3}$ to each vertex of a path or cycle. The role of higher connectivity is less clear. We do now know, however, whether there are such examples for arbitrary $k$ and for connectivity $m \leq k$. We suspect, perhaps, that even a neighborhood condition of $|N(x, y)| \geq 3 k+O(1)$ may suffice to guarantee the existence of $k$ independent chorded cycles, akin to the improvement of the result of Faudree and Gould in Theorem 2, but also that this improvement would not be easy.

In addition, we also prove the conjecture of Faudree and Gould [2]:

Theorem 2. If $G$ is a graph on at least $30 k$ vertices, such that any pair of non-adjacent vertices $x, y$ has $|N(x, y)| \geq 2 k+1$, then $G$ contains $k$ independent cycles.

This result is also sharp, in the sense that $2 k+1$ cannot be relaxed to $2 k$. Examples here include disjoint edges or trees where all leaves are adjacent to vertices of degree 2 . For $k=2$, the wheel graph serves as an example: nonadjacent vertices have neighborhood union at least four, but there are no two independent chorded cycles. Again, we do not know sharpness examples for all $k$, with all possible connectivities, so perhaps some improvements are possible.

It is possible that the constant ' 30 ' in the theorem can be improved, possibly even to 4 . Indeed, one may slightly improve this constant by being 
more careful in a few places in the proof, but it is clear that such improvements will not bring the constant all the way to 4 . Such an improvement seems to require new ideas.

\section{The proof of Theorem 1}

Before we delve into the proof, we need a definition and some examples. Since graphs with minimum degree at least 3 contain a chorded cycle, we will often be concerned with graphs that contain no subgraph with minimum degree at least 3. A graph $H$ is called 2-degenerate if it has the property that there is an ordering of vertices $v_{1}, v_{2}, \ldots, v_{h}$ of $H$ such that each vertex has at most two edges to higher labeled vertices. We call such an ordering a 2-degenerate ordering. Containing no subgraph with minimum degree at least 3 is easily seen to equivalent to being 2-degenerate.

We say that $H$ contains a $k$-tower if there is a 2-degenerate ordering of $H$, say $v_{1}, \ldots, v_{h}$, such that if $v_{i}$ is the first vertex at distance $k-1$ from $v_{1}$, then for $2 \leq j \leq i$, each intermediate vertex $v_{j}$ is adjacent to a vertex $v_{\ell}$ with $\ell<j$, that is, each $v_{j}$ has an edge to the left in the ordering. One interpretation of this definition is that there is a 2-degenerate ordering starting at some vertex $v$ such that a vertex at distance $k-1$ from $v$ can be lowered to degree 2 just by iteratively deleting vertices of degree 2 exposed by deleting $v$ and its neighbors (and iterative neighbors) while leaving other vertices of degree 2 in the graph.

An alternate definition of a $k$-tower is the following. Given a 2-degenerate ordering of $G$, say $v_{1}, \ldots, v_{h}$, then for any $i \in[h]$, one may define a rooted spanning forest $G\left[v_{1}, \ldots, v_{i}\right]$ so that the parent of $v_{k}$ is the vertex $v_{j}$ where $1 \leq j<k$ is the minimal so that $v_{j} \sim v_{k}$. If $v_{k}$ has no neighbor in $v_{1}, \ldots, v_{k-1}$, then $v_{k}$ will not have a parent (and thus will be a root), and thus it is clear that $v_{1}$ is a root. Then $H$ contains a $k$-tower if there is a 2-degenerate ordering $v_{1}, \ldots, v_{h}$, such that if $v_{i}$ is the first vertex at distance $k-1$, the spanning forest $G\left[v_{1}, \ldots, v_{i}\right]$ is connected.

One may consider this definition an analogue of having a path of length $k$ in a tree starting at a leaf and not containing any vertices of degree larger than 2. Clearly any 2-degenerate graph $H$ must contain a 1-tower (this is simply a vertex of degree at most 2 ), and if $H$ contains a $k$-tower, it clearly also contains a $(k-1)$-tower. A star is an example of a graph which only contains a 1-tower, while a path would be an $|H|$-tower. Also, a 2-degenerate graph $H$ which has a single vertex of degree 2 contains a $(\operatorname{diam}(H)+1)$ tower. 
Proof of Theorem 1. The proof is by induction on $k$ and $n=|G|$. For $k=1$, and any $n$ the result is clear: either $\delta(G) \geq 3$ and the result holds by the theorem of Finkel, or there exists a vertex $x$ with $|N(x)| \leq 2$. If $x$ is a vertex of degree $\leq 2$, since $|N(x, y)| \geq 5$ for all $y \not x$, note that $N_{G-N[x]}(y) \geq 3$. Since $\delta(G-N[x]) \geq 3$, there must be a chorded cycle in $G-N[x]$.

We now suppose that the theorem holds for all $k^{\prime}<k$ and show it holds for $k$. Let $G$ be a graph satisfying the hypothesis of Theorem 1 . If there exists a chorded 4-cycle $C$ in $G$, then note that $G-C$ satisfies the hypothesis of Theorem 1 for $k^{\prime}=k-1$. Thus, by the inductive hypothesis, there exists $k-1$ independent chorded cycles in $G-C$, and along with $C$, we have $k$ independent chorded cycles. Thus, we may assume that $G$ contains no chorded 4-cycles.

Note that $G$ satisfies the conditions for $k^{\prime}=k-1$ (even after removing 4 arbitrary vertices), and hence by the inductive hypothesis there exists $k-1$ independent chorded cycles in $G: C_{1}, C_{2}, \ldots, C_{k-1}$. Let $\mathcal{C}=\bigcup C_{i}$. We shall assume that $|\mathcal{C}|$ is minimized. Let $H=G \backslash \mathcal{C}$, and assume that $H$ has the maximum number of edges (subject to the constraint that $|\mathcal{C}|$ is minimized). If $H$ has a chorded cycle we are done, so we may assume that $H$ does not contain a chorded cycle and derive a contradiction. We note that $|H| \geq 4$. By the results of Finkel, the fact that $H$ has no chorded cycles implies that $H$ is 2-degenerate. We shall assume (subject to having the maximum number of edges) that $H$ has a $k$-tower for as large a $k$ as possible.

We make a few initial observations about $H$ :

Claim 1. If there is a $C_{i} \in \mathcal{C}$ and $x \in H$ with $\left|N_{C_{i}}(x)\right| \geq 3$, then $\left|C_{i}\right| \leq 6$.

Proof. Suppose $x$ is adjacent to $y_{1}, y_{2}, y_{3} \in C_{i}$. If the distance of $y_{i}$ and $y_{j}$ on $C$ is $>2$ for any $i, j$, then there is a shorter chorded cycle using $x$ (possibly using the edge to the other $y_{k}$ as the chord), contradicting the minimality of $|\mathcal{C}|$. Furthermore, if $y_{1}, y_{2}, y_{3}$ are three consecutive vertices in $C_{i}$, then they form a chorded 4-cycle with $x$, and we are done. The only way, then, that all of the pairwise distances of $y_{1}, y_{2}, y_{3}$ can be at most 2 is if $\left|C_{i}\right| \leq 6$.

Claim 2. If $x \not y$ with $\left|N_{H}(x, y)\right| \leq 4$ and $x, y \in H$, then there exists a chorded 6 -cycle $C_{i} \in \mathcal{C}$ such that, up to relabeling $x$ and $y$ are adjacent to $C_{i}$ in one of the following ways (see Figure 1).

Proof. Note that $\left|N_{\mathcal{C}}(x, y)\right| \geq 4 k+1-4=4 k-3$, so there exists some $C_{i} \in \mathcal{C}$ such that $\left|N_{C_{i}}(x, y)\right| \geq 5$. Without loss of generality, say, $\left|N_{C_{i}}(x)\right| \geq 3$, and hence by Claim $1,\left|C_{i}\right| \leq 6$.

If $\left|C_{i}\right|=5$, and $\left|N_{C_{i}}(x)\right| \geq 4$, then there is a chorded 4-cycle in $C_{i} \cup\{x\}$. Thus, $\left|N_{C_{i}}(x)\right|=3$, and $N_{C_{i}}(x, y)=C_{i}$. It is easy to check that however 

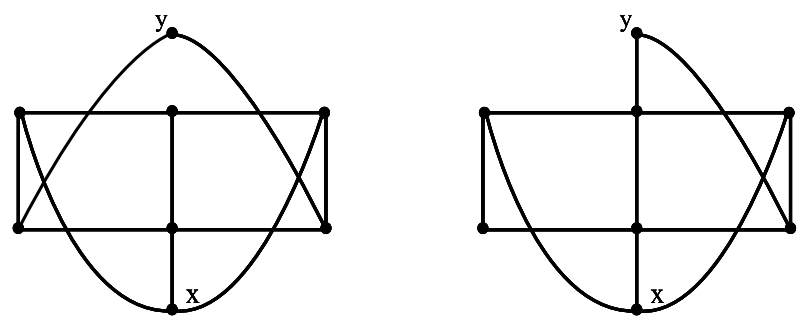

Figure 1

$x$ and $y$ send edges into $C_{i}$, they would create a chorded 4-cycle; therefore, $\left|C_{i}\right|=6$.

If the chord in $C_{i}$ connects two vertices at distance 2 on the chorded cycle, then however the 3 edges from $x$ are placed in $C_{i}$ so that the ends have pairwise distance 2 on the cycle, they admit a shorter chorded cycle. If both ends of the chord are adjacent to $x$, then there is a chorded 4-cycle. Otherwise, there is a chorded 5-cycle. Therefore, the chord bisects the $C_{6}$ as in the Figure 1. Then $\left|N_{C_{i}}(x)\right|=3$, or else there would be a chorded 5 cycle; and since the edges must be pairwise at distance 2 along the cycle, they must lie as in the Figure 1. Then $y$ has at least two neighbors in $C_{i}$ which are not neighbors of $x$, as in the Figure 1 .

A corollary of Claim 2 is the following swapping lemma, which is key to our argument.

Lemma 1 (Swapping Lemma). Suppose $x, y$ are non-adjacent vertices in $H$ with $\left|N_{H}(x, y)\right| \leq 4$. Then there exists a $C_{i} \in \mathcal{C}$ and vertices $z_{x}, z_{y} \in C_{i}$ such that $z_{x} \sim x, z_{y} \sim y$ and both $\left(C_{i}-\left\{z_{x}\right\}\right) \cup\{y\}$ and $\left(C_{i}-\left\{z_{y}\right\}\right) \cup\{x\}$ are chorded cycles. Furthermore, if $x^{\prime} \sim x$ then $z_{x} \not x^{\prime}$, likewise if $y^{\prime} \sim y$ then $z_{y} \nsim y^{\prime}$.

Proof. By Claim 2, we know the adjacencies of $x$ and $y$ to some $C_{i}$. It is easy to verify, by inspection, that a chorded cycle including, say, $x$ may be routed excluding one of the corner vertices adjacent to $y$; that corner vertex will be denoted $z_{y}$. The lemma easily follows from inspection; a typical case is as in Figure 2. Here the outer cycle of the new chorded cycle is highlighted. The assertion that $z_{x} \not x^{\prime}$ follows from the fact that if it was adjacent, it would create a chorded 5-cycle. This is easy to check; details are included as part of the proof of Lemma 2 below.

As an application of the swapping lemma, we see that (if we do not insist that the number or edges of $H$ is maximized) we may assume $H$ is connected: 


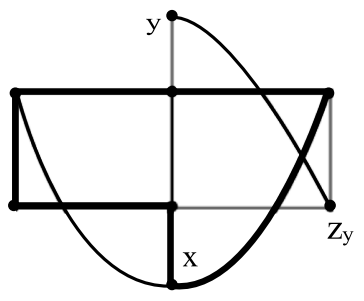

Figure 2

Suppose $H$ is such that it has a minimum number of components, and that the largest component is as large as possible. If $H$ had two components, say $X_{1}$ and $X_{2}$ where $X_{1}$ is the largest component, each would have a vertex of degree at most 2 in $H$. Suppose $x_{1} \in X_{1}$ and $x_{2} \in X_{2}$ are these vertices; then $\left|N_{H}\left(x_{1}, x_{2}\right)\right| \leq 4$. Applying the swapping lemma says that we may remove $x_{2}$ and append a vertex to $x_{1}$, which would increase the size of the largest component, a contradiction. Thus, $H$ must be connected. In particular, when the number of edges in $H$ is maximized, we know that $|E(H)| \geq|H|-1$. Also note that if the number of edges in $H$ is maximized, and we apply the swapping lemma, we know that $\left|N_{H-\{y\}}\left(z_{x}\right)\right| \leq\left|N_{H}(y)\right|$ and similarly for $z_{y}$ and $x$. The swapping lemma also implies that there are no isolated vertices in $H$.

Observe the following: Suppose $k$ is the largest integer such that $H$ has a $k$-tower. If $k \leq 2$, then $G$ has at least 2 non-adjacent vertices of degree at most 2 . Indeed, if $H$ has only a single vertex of degree $\leq 2$, then it has a $(\operatorname{diam}(G)+1)$-tower, and since $|H| \geq 4, \operatorname{diam}(H) \geq 2$. If $H$ only has two vertices of degree $\leq 2$, and they are adjacent, the same conclusion holds. $H$ cannot have just 3 pairwise adjacent vertices of degree $\leq 2$ : if $H$ had such, they would form one component of size 3 , but $|H| \geq 4$, so there must be another component which must contain a vertex of degree $\leq 2$. Furthermore, if $H$ has only a one-tower and has only two non-adjacent vertices of degree $\leq 2$, then they must both be connected to a vertex of degree at most 4 .

The following is the crux of the proof:

Lemma 2. Suppose $x, y, z$ are three vertices in $H$ with $x \sim y, y \sim z$ and $x \not z$, such that $\left|N_{\mathcal{C}}(x, z)\right|+\left|N_{\mathcal{C}}(y)\right| \geq 6 k-5$. Then $|\mathcal{C}|$ is not minimized.

Proof. Suppose that $|\mathcal{C}|$ is minimized. We shall find a shorter chorded cycle and thus, a contradiction.

By hypothesis, there exists some chorded cycle $C=C_{i} \in \mathcal{C}$ such that $\left|N_{C}(x, z)\right|+\left|N_{C}(y)\right| \geq 7$. Clearly, one of $x, y$, or $z$ sends at least 3 edges 
into $C$, so by Claim $1,|C| \leq 6$. If any one vertex sends 4 edges into $|C|$, it is clear that there is a shorter chorded cycle. We consider the other scenarios:

If $\left|N_{C}(y)\right|=3$, then $\left|N_{C}(x, z)\right| \geq 4$. First, consider $|C|=5$. Since there is only one way to fit 3 edges from a single vertex into a chorded 5 cycle without creating a shorter chorded cycle, we know the adjacencies from $y$ to $C$. If one of $x$ or $z$ (say $x$ ) also has degree 3 in $C$, then it is easily seen that $N_{C}(x)=N_{C}(y)$. Then there are clearly chorded 4-cycles. Now suppose $x$ and $z$ both have degree 2 in $C$. Suppose $b_{1}, b_{2}$ are the two vertices which lie on one side of the chord. Both $b_{1}, b_{2} \in N(y)$. One of $b_{1}, b_{2}$ (say $b_{1}$ ) is in the neighborhood of one of $x$ or $z$ (say $x$ ). But then we have a chorded 4 cycle: $x, b_{1}, b_{2}, y$ with chord $\left\{y, b_{1}\right\}$.

Thus, we may assume $|C|=6$. If $C$ is a chorded 6 -cycle with a single vertex $a$ on one side of the chord and three vertices $b_{1}, b_{2}, b_{3}$ on the other (where $b_{1}, b_{3}$ are at distance 2 from $a$, and $b_{2}$ is at distance 3 ), then $y \sim$ $\left\{a, b_{1}, b_{3}\right\}$. If $x \sim b_{1}$, say, then there is a chorded 5 cycle: $x, b_{1}, b_{2}, b_{3}, y$ with chord $\left\{y, b_{1}\right\}$. Similarly, if $x \sim a$ or $x \sim b_{3}$, or if $z$ is adjacent to any of these vertices. But $|N+C(x, z)| \geq 4$, so one of $x$ or $y$ must be adjacent to one of these vertices, a contradiction. Thus, $C$ must be the chorded 6 -cycle as in Figure 2. Suppose the vertices of the chord, arranged in order along the cycle are $x_{1}, \ldots, x_{6}$, and the chord is $\left\{x_{1}, x_{4}\right\}$. Then $y \sim\left\{x_{1}, x_{3}, x_{5}\right\}$. If $x \sim x_{1}$ (or $z \sim x_{1}$ ) then there is a chorded 5-cycle: $x, x_{1}, x_{2}, x_{3}, y$ with chord $\left\{y, x_{1}\right\}$, so $x \neq x_{1}$ and similarly $x \not\left\{x_{3}, x_{5}\right\}$. But $\left|N_{C}(x, z)\right| \geq 4$ so clearly $x$ or $z$ must be adjacent to one of those vertices. Thus, $\left|N_{C}(y)\right|<3$.

If $\left|N_{C}(y)\right|=2$, then $\left|N_{C}(x, z)\right|=5$. As argued in Claim $2,|C|=6$, and it is of the form described above: vertices $x_{1}, \ldots, x_{6}$ with chord $x_{1}, x_{4}$ where (without loss of generality) $x \sim\left\{x_{1}, x_{3}, x_{5}\right\}$. Furthermore, either $z \sim$ $\left\{x_{2}, x_{4}\right\}$ or $z \sim\left\{x_{2}, x_{6}\right\}$. First consider the case where $z \sim\left\{x_{2}, x_{4}\right\}$. Then if $y \sim x_{1}$ we have a chorded 5 -cycle $x, x_{1}, x_{2}, z, y$ with chord $\left\{y, x_{1}\right\}$. Likewise $y$ cannot be adjacent to any vertex in $N_{C}(x, z)$. But $\left|N_{C}(y)\right|=2$, so this must be the case. (Here the key property is that chorded cycles are formed using exactly one edge in $C$.) Similarly, if $\left|N_{C}(y)\right|=1$, and $\left|N_{C}(x, z)\right|=6$; the fact that $N_{C}(y) \in N_{C}(x, z)$ as $|C|=6$ will force a similar chorded 5 cycle, using $x, y, z$ and one edge in $C$, with the edge from $y$ to $C$ acting as the chord.

The remainder of the proof of Theorem 2 is to show that by the Swapping Lemma, Lemma 1, we may ensure that $H$ has vertices $x, y, z$ that satisfy the conditions of Lemma 2. This leads to a contradiction to the assumption that $H$ had no chorded cycles, completing the proof. We consider the cases:

First, suppose that $H$ has no 3 -tower. Thus, it has two non-adjacent vertices $x$ and $y$ of degree at most 2 . Let us assume that $x$ is at the start of 
the highest tower in $H$ (which by definition is at most a 2-tower.) First, let us assume that there is a 2-tower starting at $x$. We apply the swapping lemma to exchange $y$ for a $y^{\prime} \sim x$. Let $\mathcal{C}^{\prime}$ and $H^{\prime}$ denote the cycle system and new copy of $H$ after the swap. Note that $\left|N_{H^{\prime}}\left(y^{\prime}\right)\right| \leq 2$ and $y^{\prime}$ is adjacent to no neighbors of $x$ as assertions of the swapping lemma. Thus, there is a 3 -tower starting at $y^{\prime}$ and by the maximality of the tower height; this implies that $\left|N_{H^{\prime}}\left(y^{\prime}\right)\right| \leq 1$. Now since there is a 3 -tower starting at $y^{\prime}$, there is a neighbor $x^{\prime} \sim x$ such that $x^{\prime} \nsim y^{\prime}$ and $\left|N_{H^{\prime}}\left(y^{\prime}, x^{\prime}\right)\right| \leq 3$ (this is the vertex which has degree at most 2 after deletion of $y^{\prime}$ and $x$ ). Now one of $y^{\prime}$ and $x^{\prime}$ (say $x^{\prime}$ ) has $\left|N_{\mathcal{C}^{\prime}}\left(x^{\prime}\right)\right|>2 k-1$ by the neighborhood condition. Apply the swapping lemma to exchange $y^{\prime}$ for a $z \sim x^{\prime}$ (if $y^{\prime}$ is the vertex of high degree, do this in reverse). Then if $H^{\prime \prime}$ is the resulting leftover vertices $\left|N_{H}^{\prime \prime}(z)\right| \leq 2$ and $z \nsim x$. It is easy to see that $z, x^{\prime}, x$ satisfy the conditions of Lemma 2 as $\left|N_{H^{\prime \prime}}(z, x)\right| \leq 4$ so $\left|N_{\mathcal{C}^{\prime \prime}}(z, x)\right| \geq 4 k+1-4=4 k-3$ and $\left|N_{\mathcal{C}^{\prime \prime}}\left(x^{\prime}\right)\right| \geq 2 k-2$, where $\mathcal{C}^{\prime \prime}$ is the new cycle set after the swaps.

If there is a 1-tower but no 2-tower, the proof is similar. Again, we use $H^{\prime}, H^{\prime \prime}$, etc. to denote the set $H$ after swaps and $\mathcal{C}^{\prime}$ accordingly. We may apply the swapping lemma to find $y^{\prime} \sim x$, there will be a 2 -tower starting at $y^{\prime}$ and hence, $\left|N_{H^{\prime}}\left(y^{\prime}\right)\right|=1$. It is possible that, in fact, a 3-tower now starts at $y^{\prime}$. In this case, there is a vertex $x^{\prime} \sim x$ such that $\left|N_{H^{\prime}}\left(x^{\prime}, y^{\prime}\right)\right| \leq 3$ and we are in the same case as before. If not, then there is a vertex $z$ of degree at most 2 non-adjacent to $y^{\prime}$. We may again apply the swapping lemma to find a neighbor $z^{\prime} \sim y^{\prime}$ with $z^{\prime} \not x$ and $\left|N_{H^{\prime \prime}}\left(z^{\prime}\right)\right| \leq 2$. Now, $\left|N_{H^{\prime \prime}}\left(z^{\prime}, x\right)\right| \leq 4$ (as their neighborhoods contain the common vertex $y^{\prime}$ ) and we are again in the position above: one of $z^{\prime}$ or $x$ ( the swapping lemma to find a $z^{\prime \prime} \sim x$ and end up in a situation where the hypothesis of Lemma 2 are satisfied.

Thus, we may assume that $H$ has a 3-tower. Suppose $x$ is the degree (at most) 2 vertex first in the ordering defining the tower. Then there is a vertex $y$ at distance 2 from $x$ with $\left|N_{H}(x, y)\right| \leq 4$.

First, suppose that there exists such a $y$ with $\left|N_{H}(y)\right| \leq 3$, and let $z$ denote the center vertex between $x$ and $y$.

Suppose $\left|N_{H}(y)\right| \leq 3$. As $\left|N_{\mathcal{C}}(x, y)\right| \geq 4 k-3$, one of $x$ or $y$ has neighborhood in $\mathcal{C}$ of size at least $2 k-1$. If this is $x$, first consider the case where there is another vertex of degree in $H$ at most 2 anywhere else in $H$, say $w$. If so, applying the swapping lemma to find $w^{\prime} \sim x$ and taking a vertex $z^{\prime}$ of degree 3 adjacent to $x$ gives a set $w, x, z^{\prime}$ which satisfies the conditions of Lemma 2 . If this is not $x$, we consider separately the cases where $N_{H}(x)$ is connected and disconnected. If $N_{H}(x)$ is connected, we see that we can choose $z \sim y$ with $N_{H}(z)=\left\{x, y, z^{\prime}\right\}$, where $z^{\prime}$ and $z$ are the 
neighbors of $x$. Then after applying the swapping lemma to $y$ and $x$ to find $y^{\prime} \sim x$ with $\left|N_{H}\left(y^{\prime}\right)\right| \leq 3$, we may assume that $\left|N_{H-y}(z)\right|=2$. In particular $\left|N_{H}\left(z, y^{\prime}\right)\right| \leq 4$ (as the neighborhoods share $x$ ) and $z, x, y^{\prime}$ satisfy the conditions of Lemma 2. If $N_{H}(x)$ is not connected, the fact that $\left|N_{H}(y)\right| \leq 3$ says we may choose $y$ such that a middle vertex $z$ has $\left|N_{H-y}(z)\right| \leq 2$. Again, applying the swapping lemma to find $y^{\prime} \sim x$ with $\left|N_{H}\left(y^{\prime}\right)\right| \leq 3$ and $y^{\prime}, x, z$ meeting the conditions of Lemma 2. If $y$ has the high degree instead, we apply the Swapping Lemma to remove $x$ and find $x^{\prime} \sim y$, and $x^{\prime}, y, z$ will similarly meet the criteria of Lemma 2 .

If $\left|N_{H}(y)\right| \geq 4$ for all $y$ at distance 2 from $x$, there is one final case. Here $x$ is adjacent to two vertices of degree $3, x_{1}, x_{2}$, and $x_{1}$ and $x_{2}$ are both adjacent to the same two vertices $y_{1}, y_{2}$ (one of which must have degree at most 4$)$. Let $y$ the vertex of degree 4 . Note that $\left|N_{H}\left(x_{1}, x_{2}\right)\right|=3$, so one of $x_{1}, x_{2}$ (say $x_{1}$ ) has $\left|N_{\mathcal{C}}\left(x_{1}\right)\right| \geq 2 k-1$. Apply the switching lemma to $x_{1}$ and $x_{2}$ find a vertex $x_{2}^{\prime} \sim x_{1}$ with $\left|N_{H}\left(x_{2}^{\prime}\right)\right| \leq 3$ and $x_{2}^{\prime} \not x$. Then $\left|N_{H-x_{2}}(x)\right|=1$, and $\left|N_{\mathcal{C}}\left(x_{1}\right)\right| \geq 2 k-2$. Thus, $\left|N_{H}\left(x_{2}^{\prime}, x\right)\right| \leq 3$, and $x_{2}^{\prime}, x_{1}, x$ satisfy the conditions of Lemma 2 .

In all cases, we have reached a contradiction so $H$ must have contained a chorded cycle and we have completed the proof of Theorem 1.

\section{The proof of Theorem 2}

Like the proof of Theorem 1, the general strategy will be by induction on $k$, for any fixed $n$. The case $k=1$ is trivial: if $G$ has minimum degree at least 2 , clearly $G$ contains a cycle; otherwise, there is a vertex $x$ of degree 1 , and $G-N[x]$ has minimum degree $\geq 2$ by the neighborhood condition and thus, contains a cycle.

We say that a sequence $v_{1}, \ldots, v_{k}$ is a non-branching path of order $k$ in $G$, if $v_{i} \sim v_{i+1}$ and $\left|N\left(v_{i}\right)\right| \leq 2$ for all $i \leq k$.

Suppose $G$ is a graph satisfying the hypothesis of Theorem 2 for some $k$ and that the theorem has been proved for $k-1$. The hypothesis of the theorem are satisfied for $k-1$, so $G$ contains $k-1$ independent cycles $C_{1}, \ldots, C_{k-1}$. Let $\mathcal{C}=\bigcup_{i=1}^{k-1} C_{i}$ and assume that $|\mathcal{C}|$ is minimized. Let $H=$ $G-\mathcal{C}$. We assume $H$ has a minimum number of components. If $H$ contains a cycle, then we have exhibited $k$ disjoint cycles and are done, so we may assume $H$ is a forest. We prove the following swapping lemma, similar to Lemma 1:

Lemma 3. Suppose $x_{1}, x_{2}$, and $x_{3}$ are vertices in $H$ which are pairwise nonadjacent and $\left|N_{H}\left(x_{i}\right)\right| \leq 1$. Then there exists $i$ and $j$ with $i \neq j, C_{k} \subseteq \mathcal{C}$ and $z \in C_{k}$, such that $\left(C_{k}-\{z\}\right) \cup\left\{x_{i}\right\}$ is a cycle and $z \sim x_{j}$. Furthermore, $\left|N_{H}(z)\right|=1$. 
Remark. There is one key difference between this lemma and Lemma 1. In Lemma 1, we are able to choose whether to append the new vertex to $x$ or append the new vertex to $y$. Here, we have no choice: the lemma guarantees that we can swap some $x_{i}$ for some $z$ adjacent to some $x_{j}$, but we cannot guarantee (say) that we can add $x_{1}$ to a cycle and swap it out for a $z \sim x_{2}$.

To prove Lemma 3, we make the following observation, which follows immediately from the minimality of $|\mathcal{C}|$ :

Claim 3. Suppose $x \in H$ has $\left|N_{C_{i}}(x)\right| \geq 2$, then $\left|C_{i}\right| \leq 4$. Furthermore, if $\left|N_{C_{i}}(x)\right|=3$, then $\left|C_{i}\right|=3$.

Proof of Lemma 3. Note that

$$
\left|N_{\mathcal{C}}\left(x_{1}, x_{2}\right)\right|+\left|N_{\mathcal{C}}\left(x_{1}, x_{3}\right)\right|+\left|N_{\mathcal{C}}\left(x_{2}, x_{3}\right)\right| \geq 3(2 k-1)
$$

so there exists a cycle $C_{i}$ such that

$$
\left|N_{C_{i}}\left(x_{1}, x_{2}\right)\right|+\left|N_{C_{i}}\left(x_{1}, x_{3}\right)\right|+\left|N_{C_{i}}\left(x_{2}, x_{3}\right)\right| \geq 7
$$

In particular, for some pair (say $x_{1}$ and $x_{2}$ ) we have $\left|N_{C_{i}}\left(x_{1}, x_{2}\right)\right| \geq 3$. Thus, $\left|C_{i}\right| \leq 4$ by Claim 3. At least one of $x_{1}$ and $x_{2}$ have degree at least 2 into $C_{i}$, say $x_{1}$. If $\left|N_{C_{i}}\left(x_{1}\right)\right|=2$, then we will set $x_{i}=x_{1}$ and $x_{j}=x_{2}$ and one can easily check that the conditions of the theorem are satisfied, since $x_{2}$ must have a neighbor in $C_{i}$ that is not amongst the neighbors of $x_{1}$. If $\left|N_{C_{i}}\left(x_{1}\right)\right|=3$, then $\left|C_{i}\right|=3$. In this case, note that one of $x_{2}$ or $x_{3}$ must have a neighbor in $C_{i}$, otherwise (1) will not hold; if this is $x_{3}$, say, let $x_{i}=x_{1}$, and $x_{j}=x_{3}$. The other cases follow analogously.

Note that Lemma 3 implies the following:

Claim 4. H has at most 2 isolated vertices.

Proof. Otherwise, applying the swapping lemma (Lemma 3) to these vertices would reduce the number of components.

The key observation is the following:

Lemma 4. Without loss of generality, we may assume that $H$ contains either two disjoint non-branching paths of order at least 4, one of which starts at a leaf or a single non-branching path of order 8, which need not contain a leaf.

In the proof, we also need the following lemma, whose proof requires some additional setup, which we give after we complete the proof of Lemma 4. 
Lemma 5. Under the assumption that $H$ does not contain two disjoint nonbranching paths of order at least four, one of which starts at a leaf, we may assume that $|H| \geq 40$.

Proof of Lemma 4. For the purposes of this, we assume that $H$ has no isolated vertices; even after removing the isolated vertices, we decrease the size of $H$ by at most 2 by Claim 4 . We assume that $H$ cannot have a non-branching path of order 8 starting at a leaf, subject to the conditions imposed on $H$. Further, by Lemma 5, we may assume that $|H| \geq 40$. Now, we show that $H$ contains an internal non-branching path of order 8 .

Let $\ell$ denote the number of leaves in $H$ which are not in path components and $p$ denote the number of components in $H$ which are paths. Suppose $H$ is such (subject to our condition on $|\mathcal{C}|$ and that the number of components in $H$ are minimized) that $\ell+p$ is minimized. Let $L$ consist of the set of leaves in $H$, with one leaf selected from each path component. Note $|L|=\ell+p$. Note that there is a non-branching path of some order starting at each leaf. Let $\mathbf{v}=\left(v_{1}, v_{2}, \ldots,\right)$ be the vector where $v_{i}$ is the number of leaves in $L$ which are connected to a maximal non-branching path of order exactly $i$. Note, by assumption $v_{i}=0$ for $i \geq 8$. We define the weight of $\mathbf{v}$ to be:

$$
w t(\mathbf{v})=\sum_{i=1}^{\infty} v_{i}\left(1-2^{-i}\right) .
$$

Suppose that $H$ is such that (subject to its other conditions) $w t(\mathbf{v})$ is minimized. We claim that $v_{i} \leq 2$ for all $i$. If $v_{i}=3$, then applying the swapping lemma to the three vertices ending paths of order $i$ would result in a new $H^{\prime}=\left(H-\left\{x_{i}\right\}\right) \cup\{z\}$ and $\mathcal{C}^{\prime}$ where $w t\left(\mathbf{v}^{\prime}\right)<w t(\mathbf{v})$. If $\sum_{i>4} v_{i} \geq 2$, we are done. If $\sum_{i \geq 4} v_{i}=1$, then there are at most 7 leaves, and $\sum_{i} i v_{i} \leq 19$. Otherwise, there are at most 6 leaves and $\sum_{i} i v_{i} \leq 12$.

Let $J$ denote the set of vertices in $H$ of degree at least 3 (in $H$ ), and let $j=|J|$. Let $c$ denote the number of components of $H$. The vertices of $J$ divide the $n-j$ vertices of $H-J$ into

$$
\begin{aligned}
c+\sum_{v \in J}(|N(v)|-1) & =c+j+\sum_{v \in J}(|N(v)|-2) \\
& =c+j+(\ell-2(c-p))=j+\ell+2 p-c
\end{aligned}
$$

segments which are non-branching paths, some of which may be empty if there are adjacent vertices in $J$.

Of these $j+\ell+p-c$ segments, $\ell+p$ of them are attached to vertices in $L$ and these account for at most $\sum_{i} i v_{i}$ vertices. Thus, the remaining 
$\geq|H|-2-j-\sum_{i} i v_{i}$ vertices are split into $j+\ell+2 p-c-(\ell+p)=j-c+p$ segments. A crucial observation at this point is that if every component is a path component, then all vertices are in segments attached to vetices in $L$, an impossibility since $\sum_{i} i v_{i}<40$. In particular, this means that $p-c \leq-1$. If $\sum_{i \geq 4} v_{i}=1$, then there must be a non-branching path of order at least

$$
\left\lceil\frac{|H|-2-j-19}{j-c+p}\right\rceil \geq\left\lceil\frac{|H|-2-(\ell-2)-19}{(\ell-2)-c+p}\right\rceil \geq\left\lceil\frac{40-26}{4}\right\rceil \geq 4
$$

since $|H| \geq 40$. Thus, there is a non-branching path of order 4 in the interior of the forest, and along with one adjacent to a leaf, we have the two desired paths of order 4 .

If $\sum_{i \geq 4} v_{i}=0$, so that $\ell \leq 6$ and $\sum_{i} i v_{i} \leq 12$, we have a non-branching path of order at least

$$
\left\lceil\frac{|H|-2-j-12}{j-c+p}\right\rceil \geq\left\lceil\frac{|H|-2-(\ell-2)-12}{(\ell-2)-c+p}\right\rceil \geq\left\lceil\frac{40-18}{3}\right\rceil \geq 8
$$

as desired.

Before we prove Lemma 5, we need one additional claim concerning cycles with edges between them. We define the graphs $G(a, b, c, d)$ to be the disjoint union of two cycles $C_{1}$ and $C_{2}$ of order $a$ and $b$ respectively, with two vertices $v_{1}$ and $v_{2}$ at distance $d$ lying on cycle $C_{c}$ specified. $v_{1}$ is connected to all of the other cycle, while $v_{2}$ is connected to a single vertex of the other cycle. (Note that for all choices of $v_{1}, v_{2}$ and the neighbor of $v_{2}$ the resulting graphs are isomorphic.)

Lemma 6. Suppose $C_{1}$ and $C_{2}$ are two cycles with $\left|C_{1}\right|+\left|C_{2}\right| \geq 7$, and $e\left(C_{1}, C_{2}\right)$ are the edges between them. If $\left|e\left(C_{1}, C_{2}\right)\right| \geq 9$, then either $C_{1} \cup$ $C_{2} \cup e\left(C_{1}, C_{2}\right) \subseteq G\left(\left|C_{1}\right|,\left|C_{2}\right|, c, d\right)$ for some parameters $c$ and $d$, or there exist two shorter disjoint cycles.

Note that 9 here is sharp: there exist configurations of $C_{1}$ and $C_{2}$ with 8 edges between them that admit no two shorter disjoint cycles and are not contained in a $G(a, b, c, d)$.

Proof. Suppose $\left|e\left(C_{1}, C_{2}\right)\right|=9$, and let $v$ be a vertex with the largest number of edges into the other cycle. Without loss of generality, $v \in C_{1}$ and we let $d_{v}$ denote the number of vertices in $C_{2}$ incident to $v$. We show $d_{v} \geq 8$. Note that this proves the lemma: if $\left|e\left(C_{1}, C_{2}\right)\right|>9$, we consider groups of 9 edges 
at a time and it is clear that the vertex $v$ of highest degree must be the same for all groups of edges.

We fix some order on $C_{2}$. For vertices, $x, y$ in $C_{2}$, with $x \leq y$ we let $(x, y)$ and $[x, y]$ denote the open or closed intervals of vertices from $x$ to $y$. If $y \leq x$, then $[x, y]$ is the complement of $(y, x)$ and similarly for $(x, y)$. Half open intervals, e.g. $(x, y]$, are defined accordingly.

Suppose $\left|e\left(v, C_{2}\right)\right|=7$. Let $x_{1}<x_{2}<\cdots<x_{7} \in C_{2}$ denote the neighbors of $v$ in order around the cycle. Then there are two other edges from $C_{1}$ to $C_{2}$, which hit vertices $a_{1} \leq a_{2}$ in $C_{2}$ (where $a_{1}$ may be the same as $a_{2}$. Either there are three $x_{i}$ within $\left[a_{1}, a_{2}\right]$ or there are three $x_{i}$ on the outside of $\left[a_{2}, a_{1}\right]$. In either case, one can build two shorter cycles which avoids (at least) one of the three vertices to the inside (or outside) of $\left[a_{1}, a_{2}\right]$.

If $\left|e\left(v, C_{2}\right)\right|=6$, let $x_{1}<\cdots<x_{6}$ denote the vertices adjacent to $v$. Note that if $C_{1} \backslash v$ has 2 neighbors in $\left(x_{2}, x_{6}\right)$ then shorter cycles can be constructed avoiding $x_{2}$. Otherwise, $C_{1} \backslash v$ has 2 neighbors in $\left[x_{6}, x_{2}\right]$ and shorter cycles can be constructed avoiding $x_{3}$.

If $\left|e\left(v, C_{2}\right)\right|=5$, let $x_{1}<\cdots<x_{5}$ denote the vertices adjacent to $v$. If $\left[x_{5}, x_{1}\right)$ contains 2 neighbors of $C_{1} \backslash v$, then shorter cycles can be constructed avoiding $x_{2}$. Similarly, if there are two vertices in any of the intervals $\left[x_{1}, x_{3}\right)$, or $\left[x_{3}, x_{5}\right)$. But one of these must contain two elements.

If $\left|e\left(v, C_{2}\right)\right|=4$, let $x_{1}<\cdots<x_{4}$ denote the vertices adjacent to $v$. Either one of $\left[x_{4}, x_{1}\right),\left[x_{1}, x_{2}\right),\left[x_{2}, x_{3}\right)$, or $\left[x_{3}, x_{4}\right)$ contains two edges from $C_{1} \backslash v$, allowing a shorter cycle in any case or each has exactly one edge from $C_{1} \backslash v$, which also allows a shorter cycle.

If $\left|e\left(v, C_{2}\right)\right|=3$, let $x_{1}<\cdots<x_{3}$ denote the vertices adjacent to $v$. If one of $\left(x_{1}, x_{2}\right),\left(x_{2}, x_{3}\right)$ or $\left(x_{3}, x_{1}\right)$ contains two edges from $C_{1} \backslash v$, the $G$ admits a shorter cycle. Now either one of $x_{1}, x_{2}$, or $x_{3}$ has two edges from $C_{1} \backslash v$ or there is exactly one edge from $C_{1} \backslash v$ into each of $x_{1}, x_{2}, x_{3},\left(x_{1}, x_{2}\right),\left(x_{2}, x_{3}\right)$, and $\left(x_{3}, x_{1}\right)$. In the second case, each set must be non-empty, and it is easy to see that there are shorter disjoint cycles. If $x_{1}$ (say) has two edges from $C_{1} \backslash v$, then there are shorter disjoint cycles unless $\left(x_{3}, x_{1}\right)$ and $\left(x_{3}, x_{2}\right)$ are empty. This forces $x_{2}$ (say) to have two edges from $C_{1} \backslash v$, forcing $\left(x_{3}, x_{1}\right)$ to be empty, and thus, $x_{3}$ must also have two edges from $C_{1} \backslash v$. (Note that no $x_{i}$ may have 3 edges from $C_{1} \backslash v$, this would contradict the fact that $v$ has maximum degree to the other cycle.) The same argument applied in reverse (now taking one of the $x_{i}$ 's to be the vertex of degree 3 and looking at edges into $C_{2}$ ) implies that either we have two shorter cycles or, in fact, we must two triangles with a $K_{3,3}$ connecting them. This contradicts $\left|C_{1}\right|+\left|C_{2}\right| \geq 7$.

If $\left|e\left(v, C_{2}\right)\right|=2$, let $x_{1}<x_{2}$ denote the neighbors of $v$. If $\left(x_{1}, x_{2}\right)$ or $\left(x_{2}, x_{1}\right)$ have three edges from $C_{1} \backslash v$, then there are shorter disjoint cycles. 
Thus, one of $x_{1}$ or $x_{2}$ has at least 2 neighbors from $C_{1} \backslash v$. This contradicts the fact that $v$ has the maximum degree.

If $\left|e\left(v, C_{1}\right)\right|=1$, suppose $v_{1}, v_{2} \in C_{1}$ are as close together as possible such that $v_{1}, v_{2}$ have neighbors in $C_{2}$, and let $x_{1}<x_{2}$ denote their neighbors. One of $\left(x_{1}, x_{2}\right)$ or $\left(x_{2}, x_{3}\right)$ must have at least 3 neighbors in $C_{1} \backslash\left\{v_{1}, v_{2}\right\}$ and thus, there are shorter cycles.

Therefore, $\left|e\left(v, C_{2}\right)\right| \geq 8$ as desired.

Proof of Lemma 5. Suppose $|H|<40$. Then the average size of a cycle in $\mathcal{C}$ is at least $\frac{30 k-40}{k-1} \geq 20$ (since $k>1$ ). Let $C$ be a cycle in $\mathcal{C}$ of maximum order. Then $C$ has no chords, due to the minimality of $|\mathcal{C}|$. Order the vertices in $C$, and denote them $v_{0}, \ldots, v_{m}$. Let $R=\bar{C}$ denote the complement of $C$. We may assume that, without loss of generality at most two (adjacent) vertices have $\left|N_{R}(v)\right|<k-1$, and if these exist, we will assume that they are (possibly only one of) $v_{1}, v_{2}$. Pair off vertices $\left\{\left\{v_{4 i}, v_{4 i+2}\right\},\left\{v_{4 i+1}, v_{4 i+3}\right\}: i=\right.$ $0,1, \ldots,\lfloor m / 4\rfloor-1\}$. Let $L$ denote the set of vertices not paired off; there are $m(\bmod 4)$ such vertices. Then for any of these pairs $(u, v),\left|N_{C}(u, v)\right|=3$. Now consider that

$\sum_{i \leq m / 4-1}\left(\left|N_{R}\left(v_{4 i}, v_{4 i+2}\right)\right|+\mid N_{R}\left(v_{4 i+1}, v_{4 i+3}\right)\right)\left|+\sum_{v \in L}\right| N_{R}(v) \mid \geq(m+1)(k-1)$.

Note that $|e(C, H)|<20$ as if any two vertices at distance at most 9 in $H$ have neighbors in $C$, then there is a shorter cycle. Here we use the fact that $H$ is minimized with respect to $w t(\mathbf{v})$ and the condition on the nonbranching paths within $H$. Note that this is already a contradiction if $k=2$. Thus, there exists some cycle $C^{\prime} \in \mathcal{C}$, with at least $m+2$ edges between $C$ and $C^{\prime}$.

Lemma 6, along with the neighborhood condition, implies that $\left|C^{\prime}\right|=|C|$ and that a vertex in $C$ must be adjacent to every vertex in $C^{\prime}$, with one additional edge between the two. The fact that the high degree vertex is in $C$ comes from the neighborhood condition, and the fact that $\left|C^{\prime}\right|=|C|$ comes from the fact that there is a vertex in $C$ with degree $m+1$ into $C^{\prime}$, and $C$ is a maximum order cycle.

We now repeat the argument: Let $v$ be the vertex in $C$ of high degree to $C^{\prime}$. We pair off vertices of $C$ starting at the successor of $v$ and also pair off the vertices of $C^{\prime}$ to get that there must be at least $(2 m+1)(k-1)-1$ edges leaving $C \cup C^{\prime} \backslash v$; in particular, there must be third cycle $C^{\prime \prime}$ such that there are at least $2 m+1$ edges between $C$ and $C^{\prime}$ and $C^{\prime \prime}$. Applying Lemma 6 again implies $\left|C^{\prime \prime}\right|=\left|C^{\prime}\right|=|C|$ and that there is a $v^{\prime} \neq v \in C$ and a $v^{\prime \prime} \in C^{\prime}$ each of which are adjacent to at least $\left|C^{\prime \prime}\right|-1$ vertices in $\left|C^{\prime \prime}\right|$. 
But then it is clear that we can find three shorter cycles, contradicting our assumptions on $|\mathcal{C}|$. (Here, if $k=3$ the number of edges between $C \cup C^{\prime}$ and $H$ already contradict the fact that $|\mathcal{C}|$ is minimized.)

We are now ready to complete the proof of Theorem 2. By Lemma 4, we may assume that $H$ contains either two disjoint non-branching paths of order 4 or one non-branching path of order 8. First, consider the case where $H$ contains two disjoint non-branching paths $P_{1}, P_{2}$ of order 4 (one of which contains a leaf). Let $P_{1}=\left\{x_{1}, x_{2}, x_{3}, x_{4}\right\}$ and $P_{2}=\left\{y_{1}, y_{2}, y_{3}, y_{4}\right\}$, and consider the pairs of non-adjacent vertices $S=\left\{\left(x_{1}, x_{3}\right),\left(x_{2}, x_{4}\right),\left(y_{1}, y_{3}\right),\left(y_{2}, y_{4}\right)\right\}$. Then

$$
\sum_{(u, v) \in S}\left|N_{\mathcal{C}}(u, v)\right| \geq 4 \cdot(2 k-2)+1=8(k-1)+1 .
$$

Therefore, there exists a $C=C_{i}$ such that $\sum_{(u, v) \in S}\left|N_{C}(u, v)\right| \geq 9$. This implies that some vertex in one of the $P_{i}$ s has two neighbors on $C$, so $|C| \leq 4$.

First, assume that $|C|=4$. Without loss of generality, assume that

$$
\left|N_{C}\left(x_{1}, x_{3}\right)\right|+\left|N_{C}\left(x_{2}, x_{4}\right)\right| \geq\left|N_{C}\left(y_{1}, y_{3}\right)\right|+\left|N_{C}\left(y_{2}, y_{4}\right)\right| .
$$

If $\left|N_{C}\left(x_{1}, x_{3}\right)\right|+\left|N_{C}\left(x_{2}, x_{4}\right)\right| \geq 7$, then one pair, say $x_{1}$ and $x_{3}$ is adjacent to all four vertices on $C$. Then we reach a contradiction to the minimization of $|\mathcal{C}|$ as either a triangle is already formed, or $x_{2}$ or $x_{4}$ must be adjacent to some vertex on the cycle, creating a triangle. If $\left|N_{C}\left(x_{1}, x_{3}\right)\right|+\left|N_{C}\left(x_{2}, x_{4}\right)\right|=6$, then there are two vertices on $C$ which are adjacent to two vertices in $P_{1}$ (and these vertices must come from different classes, i.e. one from $\left\{x_{1}, x_{3}\right\}$ and one from $\left\{x_{2}, x_{4}\right\}$ ), and either both other vertices in $C$ are adjacent to one other vertex on $P_{1}$ or one vertex is adjacent to two vertices on $P_{1}$. Suppose first two vertices on $C$ are adjacent to two vertices of $P_{1}$, and the others are adjacent to one vertex. Then the two vertices of $C$ which are adjacent to two vertices of $P_{1}$ must be adjacent to the two end vertices of $P_{1}$ (to avoid a triangle since they must come from different classes) and must be antipodal. Label the vertices of $C$ to be (in order) $c_{1}, \ldots, c_{4}$. Then without loss of generality $x_{1}$ and $x_{4}$ are adjacent to $c_{1}$ and $c_{3}$. We are done unless both $c_{2}$ and $c_{4}$ are adjacent to one of $x_{2}$ or $x_{3}$. In this case, we must use $P_{2}$. We know that $\left|N_{C}\left(y_{1}, y_{3}\right)\right|+\left|N_{C}\left(y_{2}, y_{4}\right)\right| \geq 3$. Therefore, either some vertex in $C$ is adjacent to two vertices of $P_{2}$ (in which case it is easy to find disjoint paths), or three different vertices of $C$ are adjacent to $P_{2}$. It is easy to see here that however they are adjacent we are done, though we note that 2 edges does not suffice here: if $P_{2}$ were just adjacent to $y_{1}$ and $y_{3}$ we could not find the shorter cycles. This is the only one of these cases where we use 
the ninth edge, and hence requires care when we consider the case where we have a single path of length 8 below.

If $\left|N_{C}\left(x_{1}, x_{3}\right)\right|+\left|N_{C}\left(x_{2}, x_{4}\right)\right|=5$, then some vertex $z$ in $C$ is adjacent to two vertices of $P_{1}$, and these must be the end vertices. If $P_{2}$ is adjacent to two vertices that are not $z$, then we have two disjoint cycles. If this does not occur, $z$ occurs in the neighborhood of 2 vertices in $P_{2}$ (again, the end vertices) as in total $P_{2}$ sends three edges to $C$. But then $P_{1}$ has two edges that do not go to $z$, leading to two disjoint cycles (one involving $P_{1}$ and vertices on $C-\{z\}$ and one involving $P_{2}$ and $\{z\}$ ). We also will handle the case where $\left|N_{C}\left(x_{1}, x_{3}\right)\right|+\left|N_{C}\left(x_{2}, x_{4}\right)\right|=4$, which arises in the case where there is a non-branching path of order 8. If $\left|N_{C}\left(x_{1}, x_{3}\right)\right|+\left|N_{C}\left(x_{2}, x_{4}\right)\right|=4$, then either some vertex is adjacent to two vertices of $P_{1}$ or two vertices of $P_{2}$ which leads to the exact case above, or all vertices are adjacent to exactly one vertex in both $P_{1}$ and $P_{2}$. In either case, there are two disjoint cycles and we are done.

If $|C|=3$, then note $\left|N_{C}\left(x_{1}, x_{3}\right)\right|+\left|N_{C}\left(x_{2}, x_{4}\right)\right| \leq 6$ (again, we assume that $P_{1}$ has the larger of the neighborhoods). If this is at least 5 , two vertices on $C$ must be adjacent to two vertices of $P_{1}$, and the other vertex on $C$ must be adjacent to (at least) one vertex on $P_{1}$. If $P_{2}$ sends three edges into $C$, we are clearly done. Either two hit some vertex, and we have a cycle involving $P_{1}$ and the vertices not hit by $P_{2}$, or all three are hit and we have an analogous situation using one of the vertices that $P_{2}$ hits twice. If $P_{2}$ only sends 2 edges into $C$, then all three of the vertices of $C$ must be hit twice by $P_{1}$ and, again, we have two disjoint cycles. If $\left|N_{C}\left(x_{1}, x_{3}\right)\right|+\left|N_{C}\left(x_{2}, x_{4}\right)\right|=4$, then some vertex $z$ of $C$ must be adjacent to two vertices in $P_{1}$. Two of the edges from $P_{2}$ must be incident to vertices different than $z$, and thus we have two disjoint cycles as desired. Again, this last case cannot occur unless we are in the non-branching path of order 8 case.

Thus, we may assume we are in the case where there is a single nonbranching path of order 8; let $P=\left\{x_{1}, \ldots, x_{8}\right\}$. We can think of $P=$ $P_{1} \cup P_{2}$, where $P_{1}$ and $P_{2}$ are the first and last four vertices of $P$ respectively. The same argument as above guarantees that there is a $C$ such that $\sum_{(u, v) \in S}\left|N_{C}(u, v)\right| \geq 8$. This is where we lose something: having a leaf in our path gave us one extra edge. Note that before we used that extra edge to claim that $|C| \leq 4$ but did not use the extra edge to produce the two disjoint cycles. We showed their existence even when the (sum of the) neighborhoods of both $P_{1}$ and $P_{2}$ was of size 4 . Note either every vertex has $\left|N_{C}\left(x_{i}\right)\right|=1$ with $N_{C}\left(x_{i}\right) \neq N_{C}\left(x_{i+2}\right)$, or $|C| \leq 4$. If $|C| \leq 4$, then we can apply the above arguments, noting that we have already handled the additional cases which occur except for one noted. 
The last case with $|C| \leq 4$ is when $C=4$, and we have $C=\left\{c_{1}, c_{2}, c_{3}, c_{4}\right\}$ and $P_{1}=\left\{x_{1}, x_{2}, x_{3}, x_{4}\right\}$ where $x_{1}$ and $x_{4}$ are adjacent to both $c_{1}$ and $c_{3}$ with either $x_{2}$ or $x_{3}$ adjacent to both $c_{2}$ and $c_{4}$. Then there must be at least two edges between $P_{2}$ and $C$; if there are three then the argument above shows there exists two disjoint cycles. The only case that the above argument does not cover is if the neighbors of $P_{2}$ are $c_{1}$ and $c_{3}$. But even in this case we are done as $P_{1}$ and $P_{2}$ are adjacent: we take the cycle involving $x_{1}, c_{1}, c_{2}$, the neighbor of $c_{2}$ on $P_{1}$ (either $x_{2}$ or $x_{3}$ ) and the segment of $P_{1}$ connecting this vertex and $x_{1}$ and also the cycle involving and $x_{4}, c_{3}$, the neighbor of $c_{3}$ in $P_{2}$ and the path between $x_{4}$ and this vertex.

If every vertex has degree 1 , consider that $x_{1}$ and $x_{3}$ have two distinct neighbors on $C$. If $\left.N_{C}\left(x_{2}\right) \in\left\{N_{C}\right)\left(x_{1}\right), N_{C}\left(x_{3}\right)\right\}$, then we have a triangle, and hence, $|C|=3 \leq 4$ and we are already done. Now assume $N_{C}\left(x_{2}\right) \notin$ $\left\{N_{C}\left(x_{1}\right), N_{C}\left(x_{3}\right)\right\}$. Order the vertices along the cycle so that $N_{C}\left(x_{1}\right)<$ $N_{C}\left(x_{2}\right)<N_{C}\left(x_{3}\right)$. Then if $N_{C}\left(x_{4}\right) \notin\left[N_{C}\left(x_{1}\right), N_{C}\left(x_{2}\right)\right]$ we are done, so we may assume that $N_{C}\left(x_{4}\right)$ is in this interval.

Now consider the neighbors of $x_{i}$ for $i \geq 5$. If $N_{C}\left(x_{i}\right) \notin\left[N_{C}\left(x_{1}\right), N_{C}\left(x_{2}\right)\right]$ we are done as above. In fact, if $N_{C}\left(x_{i}\right) \in\left(N_{C}\left(x_{1}\right), N_{C}\left(x_{2}\right)\right)$ we are also done: we may use $x_{4}, x_{i}$ and the portions on the path and cycle connecting them for one cycle, using the portion of the cycle contained in $\left.\left[N_{C}\left(x_{1}\right), N_{C}\left(x_{2}\right)\right)\right]$. Then one of $N_{C}\left(x_{1}\right)$ or $N_{C}\left(x_{2}\right)$ will not be used in that cycle; we will use that vertex, along with $N_{C}\left(x_{3}\right)$ the portion connecting them in the cycle not containing $\left(N_{C}\left(x_{1}\right), N_{C}\left(x_{2}\right)\right)$ along with their neighbors on the path and the portion of the path connecting them. Thus, all vertices $x_{i}$ for $i \geq 5$ must have their neighbors be either $x_{1}$ or $x_{2}$. But then one of these vertices will have two neighbors $x_{i}$ with $i \geq 5$. This easily admits two disjoint cycles.

\section{References}

[1] K. Corrádi and A. Hajnal (1963). On the number of independent circuits in a graph. Acta Math. Acad. Sci. Hungar. 14 423-443. MR0200185

[2] J. Faudree and R. J. Gould (2005). Neighborhood unions and independent cycles. Ars Combinatoria 76 29-31. MR2152745

[3] D. Finkel (2008). On the number of independent chorded cycles in a graph. Discrete Mathematics 22 5265-5268. MR2450460

[4] P. Justesen (1989). On independent circuits in finite graphs and a conjecture of Erdős and Posa. Annals of Discrete Math. 41 299-306. MR0976008 
[5] H. Wang (1999). On the maximum number of independent cycles in a graph. Discrete Math. 205(1-3) 183-190. MR1703268

Ronald J. Gould

Department of Mathematics and Computer Science

EMORY UNIVERSITY

AtLanta, GA 30322

USA

E-mail address: rg@mathcs. emory. edu

Kazuhide Hirohata

Department of Electronic and Computer Engineering

Ibaraki National College of Technology

IBARAKI

JAPAN

E-mail address: hirohata@ece.ibaraki-ct.ac.jp

Paul Horn

Department of Mathematics

HARVARD UNIVERSITY

CAMBridge, MA 02138

USA

E-mail address: phorn@math.harvard.edu

Received September 22, 2012 